\title{
POLYHEDRAL SURFACES AND DETERMINANT OF LAPLACIAN
}

\author{
ALEXEY KOKOTOV
}

(Communicated by Varghese Mathai)

\begin{abstract}
An explicit formula for the determinant of the Laplacian on a compact polyhedral surface of genus $g>1$ is found. This formula generalizes previously known results for flat surfaces with trivial holonomy and compact polyhedral tori.
\end{abstract}

\section{INTRODUCTION}

The main goal of the present paper is to study the determinant of the Laplacian (acting in the trivial line bundle) as a functional on the space of Riemann surfaces with conformal flat conical metrics (polyhedral surfaces).

In [10] the determinant of the Laplacian was studied as a functional

$$
\mathcal{H}_{g}\left(k_{1}, \ldots, k_{M}\right) \ni(\mathcal{X}, \omega) \mapsto \operatorname{det} \Delta^{|\omega|^{2}}
$$

on the space $\mathcal{H}_{g}\left(k_{1}, \ldots, k_{M}\right)$ of equivalence classes of pairs $(\mathcal{X}, \omega)$, where $\mathcal{X}$ is a compact Riemann surface of genus $g$ and $\omega$ is a holomorphic one-form (an Abelian differential) with $M$ zeros of multiplicities $k_{1}, \ldots, k_{M}$. Here $\operatorname{det} \Delta^{|\omega|^{2}}$ stands for the determinant of the Laplacian in the flat metric $|\omega|^{2}$ having conical singularities at the zeros of $\omega$. The flat conical metrics $|\omega|^{2}$ considered in [10] are very special: the divisor of the conical points of this metric is not arbitrary (it belongs to the canonical class of divisors) and the conical angles at the conical points are integer multiples of $2 \pi$. Later, in [8], this restrictive condition has been eliminated in the case of polyhedral surfaces of genus one (it should be noted that the case of genus zero was studied in [2]).

Here we generalize the results of [10, 8], 2] to the case of polyhedral surfaces of an arbitrary genus. The main result of the paper, the explicit formula for the determinant, is given by equation (30) below. We derive it as a simple consequence of the results from [10] and an analog of the Polyakov formula for the ratio of determinants of Laplacians corresponding to two conformally equivalent flat conical metrics.

\section{Flat conical metrics on Riemann surfaces}

2.1. Troyanov theorem. Let $\sum_{k=1}^{N} b_{k} P_{k}$ be a (generalized; i.e., the coefficients $b_{k}$ are not necessarily integers) divisor on a compact Riemann surface $\mathcal{X}$ of genus g. Also let $\sum_{k=1}^{N} b_{k}=2 g-2$. Then, according to Troyanov's theorem (see [18]), there exists a (unique up to a rescaling) conformal (i.e. giving rise to a complex

Received by the editors July 5, 2010.

2010 Mathematics Subject Classification. Primary 58J52; Secondary 14H15, 30F10, 32 G15.

(C)2012 American Mathematical Society Reverts to public domain 28 years from publication 
structure which coincides with that of $\mathcal{X}$ ) flat metric $\mathbf{m}$ on $\mathcal{X}$ which is smooth in $\mathcal{X} \backslash\left\{P_{1}, \ldots, P_{N}\right\}$ and has simple singularities of order $b_{k}$ at $P_{k}$. The latter means that in a vicinity of $P_{k}$ the metric $\mathbf{m}$ can be represented in the form

$$
\mathbf{m}=e^{u(z, \bar{z})}|z|^{2 b_{k}}|d z|^{2},
$$

where $z$ is a conformal coordinate and $u$ is a smooth real-valued function. In particular, if $b_{k}>-1$ the point $P_{k}$ is conical with conical angle $\beta_{k}=2 \pi\left(b_{k}+1\right)$.

Let us outline a short proof of this theorem assuming for simplicity that all the divisors $g P_{k}, k=1, \ldots, N$ are nonspecial (this means that there is no meromorphic function with the only pole at $P_{k}$ of multiplicity $\left.\leq g\right)$ and $g>1$.

Fix a canonical basis of cycles $\left\{\mathbf{a}_{k}, \mathbf{b}_{k}\right\}_{k=1}^{g}$ on $\mathcal{X}$ and let $E(P, Q)$ be the primeform (see [5]). Let $\left\{v_{k}\right\}_{k=1}^{g}$ be the basis of holomorphic normalized differentials and denote by $\mathbb{B}$ the corresponding matrix of $\mathbf{b}$-periods. Also let $K^{P_{0}}$ be the vector of Riemann constants corresponding to a base point $P_{0}$. Introduce real vectors $\alpha, \beta$ via $K^{P_{0}}=\mathbb{B} \alpha+\beta$ and let $\Theta\left[\begin{array}{l}\alpha \\ \beta\end{array}\right](z \mid \mathbb{B})$ be the Riemann theta-function with characteristics $\alpha, \beta$. Assume that the divisor $g P_{0}$ is nonspecial (i.e. the point $P_{0}$ is not a Weierstrass point). Using the Riemann theorem on the zeros of the theta-function it is easy to show that (cf. [6], p. 32)

$$
\mathcal{F}_{P_{0}}(\cdot)=\frac{\Theta^{2}\left[\begin{array}{l}
\alpha \\
\beta
\end{array}\right]\left(\int_{.^{P_{0}}}\left(v_{1}, \ldots, v_{g}\right)^{t} \mid \mathbb{B}\right)}{E^{2}\left(\cdot, P_{0}\right)}
$$

is a Prym differential with unitary multipliers $\exp \left\{-4 \pi i \alpha_{k}\right\}, \exp \left\{4 \pi i \beta_{k}\right\}$ along the basic cycles $a_{k}, b_{k}$ respectively with a single zero of multiplicity $2 g-2$ at $P_{0}$. (Notice that if the divisor $g P_{0}$ is special, then $\mathcal{F}_{P_{0}} \equiv 0$.) Now the Troyanov metric is explicitly given by

$$
\mathbf{m}=\prod_{k=1}^{N}\left|\mathcal{F}_{P_{k}}\right|^{\frac{2 b_{k}}{2 g-2}} .
$$

Remark 1. The case when some conical point $P_{k}$ is a Weierstrass point is a bit more technical and we do not consider it here. Notice only that if the Riemann surface $\mathcal{X}$ is hyperelliptic, then this situation is even more simple than the generic one. Namely, let the corresponding algebraic curve be given by the equation $y^{2}=$ $\prod_{i=1}^{2 g+2}\left(x-x_{i}\right)$. Then the Weierstrass point $P_{k}$ coincides with one of the branch points (say, $\left(x_{j}, 0\right)$ ) and the Prym differential $\mathcal{F}_{P_{k}}$ in (2) should be replaced by the holomorphic one-form $y^{-1}\left(x-x_{j}\right)^{g-1} d x$ which has the single zero at $P_{k}$ of multiplicity $2 g-2$.

2.2. Distinguished local parameter. In a vicinity of a conical point the flat metric (11) takes the form

$$
\mathbf{m}=|g(z)|^{2}|z|^{2 b}|d z|^{2}
$$

with some holomorphic function $g$ such that $g(0) \neq 0$. It is easy to show (see, e.g., [18], Proposition 2) that there exists a holomorphic change of variable $z=z(x)$ such that in the local parameter $x$,

$$
\mathbf{m}=|x|^{2 b}|d x|^{2} .
$$

We shall call the parameter $x$ (unique up to a constant factor $c,|c|=1$ ) distinguished. In case $b>-1$ the existence of the distinguished parameter means that 
in a vicinity of a conical point the surface $\mathcal{X}$ is isometric to the standard cone with conical angle $\beta=2 \pi(b+1)$.

In [18] it is proved that any compact Riemann surface with flat conformal conical metric admits a proper triangulation (i.e., each conical point is a vertex of some triangle of the triangulation). This means that any compact Riemann surface with a flat conical metric is a Euclidean polyhedral surface, i.e., can be glued from Euclidean triangles. On the other hand any compact Euclidean oriented polyhedral surface gives rise to a Riemann surface with a flat conical metric. Therefore, from now on we do not distinguish between compact (oriented) Euclidean polyhedral surfaces and Riemann surfaces with conformal flat conical metrics.

\section{LAPLACIANS ON POLYHEDRAL SURFACES}

Let $\mathcal{X}$ be a compact polyhedral surface with vertices (conical points) $P_{1}, \ldots, P_{N}$. The Laplacian $\Delta$ corresponding to the natural flat conical metric on $\mathcal{X}$ with domain $C_{0}^{\infty}\left(\mathcal{X} \backslash\left\{P_{1}, \ldots, P_{N}\right\}\right)$ is not essentially selfadjoint and one has to choose one of its selfadjoint extensions. From now on we denote by $\Delta$ the Friedrichs extension of the Laplacian on the polyhedral surface $\mathcal{X}$; other extensions will not be considered here.

\subsection{Determinant of the Laplacian and analytic surgery.}

Theorem 1 (see 4], 7], 10). Let $\mathcal{X}$ be a compact polyhedral surface with vertices $P_{1}, \ldots, P_{N}$ of conical angles $\beta_{1}, \ldots, \beta_{N}$. Let $\Delta$ be the Friedrichs extension of the Laplacian defined on functions from $C_{0}^{\infty}\left(\mathcal{X} \backslash\left\{P_{1}, \ldots, P_{N}\right\}\right)$. Then

(1) The spectrum of the operator $\Delta$ is discrete; all the eigenvalues of $\Delta$ have finite multiplicity.

(2) Introduce the operator $\zeta$-function

$$
\zeta_{\Delta}(s)=\sum_{\lambda_{k}>0} \frac{1}{\lambda_{k}^{s}}
$$

where the summation goes over all strictly positive eigenvalues $\lambda_{k}$ of the operator $-\Delta$ (counting multiplicities). One has the equality

$$
\zeta_{\Delta}(s)=\frac{1}{\Gamma(s)}\left\{\frac{\operatorname{Area}(\mathcal{X})}{4 \pi(s-1)}+\left[\frac{1}{12} \sum_{k=1}^{N}\left\{\frac{2 \pi}{\beta_{k}}-\frac{\beta_{k}}{2 \pi}\right\}-1\right] \frac{1}{s}+e(s)\right\},
$$

where $e(s)$ is an entire function.

Theorem 1 opens a way to define the determinant, $\operatorname{det}^{*} \Delta$, of the Laplacian on a compact polyhedral surface. Namely since $\zeta_{\Delta}$ is regular at $s=0$, one can define the $\zeta$-regularized determinant of the Laplacian via the usual $\zeta$-regularization (cf. [16]):

$$
\operatorname{det}^{*} \Delta:=\exp \left\{-\zeta_{\Delta}^{\prime}(0)\right\}
$$

Remark 2. In what follows the symbol det* is used to denote the (modified) zetaregularized determinant of an operator with zero modes. The symbol det refers to the zeta-regularized determinant of an operator without zero modes. 
Equation (4) and the relation $\sum_{k=1}^{N} b_{k}=2 g-2, b_{k}=\frac{\beta_{k}}{2 \pi}-1$ yield

$$
\zeta_{\Delta}(0)=\frac{1}{12} \sum_{k=1}^{N}\left\{\frac{2 \pi}{\beta_{k}}-\frac{\beta_{k}}{2 \pi}\right\}-1=\left(\frac{\chi(\mathcal{X})}{6}-1\right)+\frac{1}{12} \sum_{k=1}^{N}\left\{\frac{2 \pi}{\beta_{k}}+\frac{\beta_{k}}{2 \pi}-2\right\}
$$

where $\chi(\mathcal{X})=2-2 g$ is the Euler characteristic of $\mathcal{X}$.

It should be noted that the term $\frac{\chi(\mathcal{X})}{6}-1$ at the right-hand side of ([6) coincides with the value at zero of the operator $\zeta$-function of the Laplacian corresponding to an arbitrary smooth metric on $\mathcal{X}$ (see, e.g., [14, formula (5a) or [15]).

Let $\mathbf{m}$ and $\tilde{\mathbf{m}}=\kappa \mathbf{m}, \kappa>0$ be two homothetic flat metrics with the same conical points with conical angles $\beta_{1}, \ldots, \beta_{N}$. Then (3), (5) and (6) imply the following rescaling property of the conical Laplacian:

$$
\operatorname{det}^{*} \Delta^{\tilde{\mathbf{m}}}=\kappa^{-\left(\frac{\chi(\mathcal{X})}{6}-1\right)-\frac{1}{12} \sum_{k=1}^{N}\left\{\frac{2 \pi}{\beta_{k}}+\frac{\beta_{k}}{2 \pi}-2\right\}} \operatorname{det}^{*} \Delta^{\mathbf{m}} .
$$

Let $\mathbf{m}$ be an arbitrary smooth metric on $\mathcal{X}$ and denote by $\Delta^{\mathbf{m}}$ the corresponding Laplacian. Consider $N$ nonoverlapping connected and simply connected domains $D_{1}, \ldots, D_{N} \subset \mathcal{X}$ bounded by closed curves $\gamma_{1}, \ldots, \gamma_{N}$ and introduce also the domain $\Sigma=\mathcal{X} \backslash \bigcup_{k=1}^{N} D_{k}$ and the contour $\Gamma=\bigcup_{k=1}^{N} \gamma_{k}$.

Define the Neumann jump operator $R: C^{\infty}(\Gamma) \rightarrow C^{\infty}(\Gamma)$ by

$$
\left.R(f)\right|_{\gamma_{k}}=\partial_{\nu}\left(V_{k}^{-}-V_{k}^{+}\right),
$$

where $\nu$ is the outward normal to $\gamma_{k}=\partial D_{k}$, the functions $V_{k}^{-}$and $V^{+}$are the solutions of the boundary value problems $\Delta^{\mathbf{m}} V_{k}^{-}=0$ in $D_{k},\left.V^{-}\right|_{\partial D_{k}}=f$ and $\Delta^{\mathbf{m}} V^{+}=0$ in $\Sigma,\left.V^{+}\right|_{\Gamma}=f$. The Neumann jump operator is an elliptic pseudodifferential operator of order 1 , and it is known that one can define its determinant via the standard $\zeta$-regularization.

Let $\left(\Delta^{\mathbf{m}} \mid D_{k}\right)$ and $\left(\Delta^{\mathbf{m}} \mid \Sigma\right)$ be the operators of the Dirichlet boundary problem for $\Delta^{\mathbf{m}}$ in domains $D_{k}$ and $\Sigma$ respectively. The determinants of these operators also can be defined via $\zeta$-regularization.

Due to Theorem $B^{*}$ from [3], we have

$$
\operatorname{det}^{*} \Delta^{\mathbf{m}}=\left\{\prod_{k=1}^{N} \operatorname{det}\left(\Delta^{\mathbf{m}} \mid D_{k}\right)\right\} \operatorname{det}\left(\Delta^{\mathbf{m}} \mid \Sigma\right) \operatorname{det}^{*} R\{\operatorname{Area}(\mathcal{X}, \mathbf{m})\}\{l(\Gamma)\}^{-1},
$$

where $l(\Gamma)$ is the length of the contour $\Gamma$ in the metric $\mathbf{m}$.

An analogous statement holds for the flat conical metric. Namely let $\mathcal{X}$ be a compact polyhedral surface with vertices $P_{1}, \ldots, P_{N}$ and $g$ be a corresponding flat metric with conical singularities. Choose the domains $D_{k}, k=1, \ldots, N$ that are nonoverlapping disks centered at $P_{k}$ and let $\left(\Delta \mid D_{k}\right)$ be the Friedrichs extension of the Laplacian with domain $C_{0}^{\infty}\left(D_{k} \backslash P_{k}\right)$ in $L_{2}\left(D_{k}\right)$. Then formula (8) is still valid with $\Delta^{\mathbf{m}}=\Delta$ (cf. [11] or see [13] for a more general result).

3.2. Polyakov and Alvarez formulas. We state Polyakov's formula in the form given in $\left([6]\right.$, p. 62). Let $\mathbf{m}_{1}=\rho_{1}^{-2}(z, \bar{z}) \widehat{d z}$ and $\mathbf{m}_{2}=\rho_{2}^{-2}(z, \bar{z}) \widehat{d z}$ be two smooth conformal metrics on $\mathcal{X}$ and let $\operatorname{det} \Delta^{\mathbf{m}_{1}}$ and $\operatorname{det} \Delta^{\mathbf{m}_{2}}$ be the determinants of the corresponding Laplacians (defined via the standard Ray-Singer regularization). Then

$$
\log \frac{\operatorname{det}^{*} \Delta^{\mathbf{m}_{2}}}{\operatorname{det}^{*} \Delta^{\mathbf{m}_{1}}}=\log \frac{\operatorname{Area}\left(\mathcal{X}, \mathbf{m}_{2}\right)}{\operatorname{Area}\left(\mathcal{X}, \mathbf{m}_{1}\right)}+\frac{1}{3 \pi} \int_{\mathcal{X}} \log \frac{\rho_{2}}{\rho_{1}} \partial_{z \bar{z}}^{2} \log \left(\rho_{2} \rho_{1}\right) \widehat{d z} .
$$


We need also the following version (belonging to Alvarez) of (9) for surfaces with boundary ([1]; see also [15]). Let $\mathcal{X}$ be a Riemann surface with smooth boundary $\partial X$ and let $\left(\Delta^{\mathbf{m}_{1}} \mid \mathcal{X}\right)$ and $\left(\Delta^{\mathbf{m}_{2}} \mid \mathcal{X}\right)$ be the operators of the Dirichlet boundary problems for $\Delta^{\mathbf{m}_{1}}$ and $\Delta^{\mathbf{m}_{2}}$. Then

$$
\begin{gathered}
\log \frac{\operatorname{det}\left(\Delta^{\mathbf{m}_{2}} \mid \mathcal{X}\right)}{\operatorname{det}\left(\Delta^{\mathbf{m}_{1}} \mid \mathcal{X}\right)}=\frac{1}{3 \pi} \int_{\mathcal{X}} \log \frac{\rho_{2}}{\rho_{1}} \partial_{z \bar{z}}^{2} \log \left(\rho_{2} \rho_{1}\right) \widehat{d z} \\
-\frac{1}{12 \pi} \int_{\partial \mathcal{X}} \log \frac{\rho_{2}}{\rho_{1}} \partial_{n} \log \frac{\rho_{2}}{\rho_{1}} d s_{1}-\frac{1}{6 \pi} \int_{\partial \mathcal{X}} k_{1} \log \left(\frac{\rho_{2}}{\rho_{1}}\right) d s_{1}+\frac{1}{4 \pi} \int_{\partial \mathcal{X}} \partial_{n} \log \frac{\rho_{2}}{\rho_{1}} d s_{1},
\end{gathered}
$$

where $k_{j}$ and $d s_{j}$ are the geodesic curvature of $\partial X$ and the length element of $\partial \mathcal{X}$ corresponding to the metric $\mathbf{m}_{j} ; n$ is the exterior normal.

\subsection{Analog of Polyakov's formula for a pair of conformally equivalent flat conical metrics.}

Proposition 1. 1 Let $a_{1}, \ldots, a_{N}$ and $b_{1}, \ldots, b_{M}$ be real numbers which are greater than -1 and satisfy $a_{1}+\cdots+a_{N}=b_{1}+\cdots+b_{M}=2 g-2$. Let $\mathbf{m}_{1}$ and $\mathbf{m}_{2}$ be two conformally equivalent flat conical metrics on $\mathcal{X}$; let $\mathbf{m}_{1}$ have conical singularities at $P_{1}, \ldots, P_{N} \in \mathcal{X}$ with conical angles $2 \pi\left(a_{1}+1\right), \ldots, 2 \pi\left(a_{N}+1\right)$ and $\mathbf{m}_{2}$ have conical singularities at $Q_{1}, \ldots, Q_{M} \in L$ with conical angles $2 \pi\left(b_{1}+1\right), \ldots, 2 \pi\left(b_{M}+1\right)$. Assume also that the sets $\left\{P_{1}, \ldots, P_{N}\right\}$ and $\left\{Q_{1}, \ldots, Q_{M}\right\}$ do not intersect.

Let $x_{k}$ be a distinguished local parameter for $\mathbf{m}_{1}$ near $P_{k}$ and $y_{l}$ be a distinguished local parameter for $\mathbf{m}_{2}$ near $Q_{l}$ (we omit the argument $t$ ).

Introduce the functions $f_{k}, g_{l}$ and the complex numbers $\mathbf{f}_{\mathbf{k}}, \mathbf{g}_{\mathbf{l}}$ by

$$
\begin{array}{ccc}
\mathbf{m}_{2}=\left|f_{k}\left(x_{k}\right)\right|^{2}\left|d x_{k}\right|^{2} & \text { near } \quad P_{k} ; & \mathbf{f}_{\mathbf{k}}:=f_{k}(0), \\
\mathbf{m}_{1}=\left|g_{l}\left(y_{l}\right)\right|^{2}\left|d y_{l}\right|^{2} & \text { near } \quad Q_{l} ; \quad \mathbf{g}_{\mathbf{l}}:=g_{l}(0) .
\end{array}
$$

Then the following equality holds:

$$
\frac{\operatorname{det}^{*} \Delta^{\mathbf{m}_{1}}}{\operatorname{det}^{*} \Delta^{\mathbf{m}_{2}}}=\frac{\prod_{k=1}^{N} C\left(a_{k}\right)}{\prod_{l=1}^{M} C\left(b_{l}\right)} \frac{\operatorname{Area}\left(\mathcal{X}, \mathbf{m}_{1}\right)}{\operatorname{Area}\left(\mathcal{X}, \mathbf{m}_{2}\right)} \frac{\prod_{l=1}^{M}\left|\mathbf{g}_{\mathbf{l}}\right|^{b_{l} / 6}}{\prod_{k=1}^{N}\left|\mathbf{f}_{\mathbf{k}}\right|^{a_{k} / 6}},
$$

where $C\left(a_{k}\right)$ (resp. $C\left(b_{l}\right)$ ) is the ratio of two determinants: the determinant of the Laplace operator with Dirichlet boundary conditions on the right circular cone with slant height $\frac{1}{a_{k}+1}\left(\right.$ resp. $\left.\frac{1}{b_{l}+1}\right)$ and the angle $2 \pi\left(a_{k}+1\right)$ (resp. $2 \pi\left(b_{l}+1\right)$ ) around the apex and the determinant of the standard Laplacian $\Delta^{|d x|^{2}}=4 \partial_{x} \bar{\partial}_{x}$ with Dirichlet boundary conditions in the unit disk $D(1)=\{|x| \leq 1\}$.

Proof. Take $\epsilon>0$ and introduce the disks $D_{k}(\epsilon), k=1, \ldots, M+N$ centered at the points $P_{1}, \ldots, P_{N}, Q_{1}, \ldots, Q_{M} ; D_{k}(\epsilon)=\left\{\left|x_{k}\right| \leq \epsilon\right\}$ for $k=1, \ldots, N$ and $D_{N+l}(\epsilon)=\left\{\left|y_{l}\right| \leq \epsilon\right\}$ for $l=1, \ldots, M$. Let $h_{k}: \overline{\mathbb{R}}_{+} \rightarrow \mathbb{R}, k=1, \ldots, N+M$ be smooth positive functions such that

(1)

$$
\int_{0}^{1} h_{k}^{2}(r) r d r=\left\{\begin{array}{l}
\int_{0}^{1} r^{2 a_{k}+1} d r=\frac{1}{2 a_{k}+2}, \quad \text { if } k=1, \ldots, N \\
\int_{0}^{1} r^{2 b_{l}+1} d r=\frac{1}{2 b_{l}+2}, \quad \text { if } k=N+l, l=1, \ldots, M
\end{array}\right.
$$

\footnotetext{
${ }^{1}$ The author thanks G. Carron and L. Hillairet for a significant improvement of the preliminary version of this proposition (the idea of introducing constants $C\left(a_{k}\right)$ ) and for pointing out to him reference [17.
} 
(2)

$$
h_{k}(r)=\left\{\begin{array}{l}
r^{a_{k}} \text { for } r \geq 1 \text { if } k=1, \ldots, N \\
r^{b_{l}} \text { for } r \geq 1 \text { if } k=N+l, l=1, \ldots, M .
\end{array}\right.
$$

Define two families of smooth metrics $\mathbf{m}_{1}^{\epsilon}, \mathbf{m}_{2}^{\epsilon}$ on $\mathcal{X}$ via

$$
\begin{gathered}
\mathbf{m}_{1}^{\epsilon}(z)=\left\{\begin{array}{l}
\epsilon^{2 a_{k}} h_{k}^{2}\left(\left|x_{k}\right| / \epsilon\right)\left|d x_{k}\right|^{2}, \quad z \in D_{k}(\epsilon), \quad k=1, \ldots, N, \\
\mathbf{m}_{1}(z), \quad z \in \mathcal{X} \backslash \bigcup_{k=1}^{N} D_{k}(\epsilon),
\end{array}\right. \\
\mathbf{m}_{2}^{\epsilon}(z)=\left\{\begin{array}{l}
\epsilon^{2 b_{l}} h_{N+l}^{2}\left(\left|y_{l}\right| / \epsilon\right)\left|d y_{l}\right|^{2}, \quad z \in D_{N+l}(\epsilon), \quad l=1, \ldots, M, \\
\mathbf{m}_{2}(z), \quad z \in \mathcal{X} \backslash \bigcup_{l=1}^{M} D_{N+l}(\epsilon) .
\end{array}\right.
\end{gathered}
$$

The metrics $\mathbf{m}_{1,2}^{\epsilon}$ converge to $\mathbf{m}_{1,2}$ as $\epsilon \rightarrow 0$ and

$$
\operatorname{Area}\left(\mathcal{X}, \mathbf{m}_{1,2}^{\epsilon}\right)=\operatorname{Area}\left(\mathcal{X}, \mathbf{m}_{1,2}\right) \text {. }
$$

Due to the analytic surgery formulas one has

$$
\operatorname{det}^{*} \Delta^{\mathbf{m}_{1}}=\left\{\prod_{k=1}^{N} \operatorname{det}\left(\Delta^{\mathbf{m}_{1}} \mid D_{k}(\epsilon)\right)\right\} \operatorname{det}\left(\Delta^{\mathbf{m}_{1}} \mid \Sigma\right) \operatorname{det}^{*} R\left\{\operatorname{Area}\left(\mathcal{X}, \mathbf{m}_{1}\right)\right\}\{l(\Gamma)\}^{-1},
$$

$$
\operatorname{det}^{*} \Delta^{\mathbf{m}_{1}^{\epsilon}}=\left\{\prod_{k=1}^{N} \operatorname{det}\left(\Delta^{\mathbf{m}_{1}^{\epsilon}} \mid D_{k}(\epsilon)\right)\right\} \operatorname{det}\left(\Delta^{\mathbf{m}_{1}^{\epsilon}} \mid \Sigma\right) \operatorname{det}^{*} R\left\{\operatorname{Area}\left(\mathcal{X}, \mathbf{m}_{1}^{\epsilon}\right)\right\}\{l(\Gamma)\}^{-1},
$$

with $\Sigma=\mathcal{X} \backslash \bigcup_{k=1}^{N} D_{k}(\epsilon)$ and analogous expressions for $\operatorname{det}^{*} \Delta^{\mathbf{m}_{1}}$ and $\operatorname{det}^{*} \Delta^{\mathbf{m}_{1}^{\epsilon}}$

Using these relations and the fact that the quantity $\operatorname{det}^{*} R / l(\Gamma)$ is a conformal invariant, we obtain that

$$
\frac{\operatorname{det}^{*} \Delta^{\mathbf{m}_{1}}}{\operatorname{det}^{*} \Delta^{\mathbf{m}_{2}}}=\frac{\left\{\prod_{k=1}^{N}\left(\Delta^{\mathbf{m}_{1}} \mid D_{k}(\epsilon)\right)\right\}}{\left\{\prod_{l=1}^{M}\left(\Delta^{\mathbf{m}_{2}} \mid D_{N+l}(\epsilon)\right)\right\}} \frac{\left\{\prod_{l=1}^{M}\left(\Delta^{\mathbf{m}_{2}^{\epsilon}} \mid D_{N+l}(\epsilon)\right)\right\}}{\left\{\prod_{k=1}^{N}\left(\Delta^{\mathbf{m}_{1}^{\epsilon}} \mid D_{k}(\epsilon)\right)\right\}} \frac{\operatorname{det}^{*} \Delta^{\mathbf{m}_{1}^{\epsilon}}}{\operatorname{det}^{*} \Delta^{\mathbf{m}_{2}^{\epsilon}}} .
$$

Applying Polyakov's formula to the last term, $\frac{\operatorname{det}^{*} \Delta^{\mathbf{m}_{1}^{\epsilon}}}{\operatorname{det}^{*} \Delta^{\mathbf{m}_{2}^{\epsilon}}}$, in the right-hand side of (14), one rewrites it as

$$
\begin{aligned}
& \frac{\operatorname{Area}\left(\mathcal{X}, \mathbf{m}_{1}\right)}{\operatorname{Area}\left(\mathcal{X}, \mathbf{m}_{2}\right)} \exp \left\{\frac{1}{3 \pi} \sum_{k=1}^{N}\right.\left(\int_{D_{k}(\epsilon)}\left(\log H_{k}\right)_{x_{k} \bar{x}_{k}} \log \left|f_{k}\right| \widehat{d x_{k}}\right. \\
&\left.+\int_{D_{k}(\epsilon)}\left(\log H_{k}\right)_{x_{k} \bar{x}_{k}} \log H_{k} \widehat{d x_{k}}\right)-\frac{1}{3 \pi} \sum_{l=1}^{M}\left(\int_{D_{N+l}(\epsilon)}\left(\log H_{N+l}\right)_{y_{l} \bar{y}_{l}} \log \left|g_{l}\right| \widehat{d y_{l}}\right. \\
&\left.\left.+\int_{D_{N+l}(\epsilon)}\left(\log H_{N+l}\right)_{y_{l} \bar{y}_{l}} \log H_{N+l} \widehat{d y_{l}}\right)\right\},
\end{aligned}
$$

where $H_{k}\left(x_{k}\right)=\epsilon^{-a_{k}} h_{k}^{-1}\left(\left|x_{k}\right| / \epsilon\right), k=1, \ldots, N$ and $H_{N+l}\left(y_{l}\right)=\epsilon^{-b_{l}} h_{N+l}^{-1}\left(\left|y_{l}\right| / \epsilon\right)$, $l=1, \ldots, M$. Notice that for $k=1, \ldots, N$ the function $H_{k}$ coincides with $\left|x_{k}\right|^{-a_{k}}$ 
in a vicinity of the circle $\left\{\left|x_{k}\right|=\epsilon\right\}$ and the Green formula implies that

$$
\begin{aligned}
& \int_{D_{k}(\epsilon)}\left(\log H_{k}\right)_{x_{k} \bar{x}_{k}} \log \left|f_{k}\right| \widehat{d x_{k}}=\frac{i}{2}\left\{\oint_{\left|x_{k}\right|=\epsilon}\left(\log \left|x_{k}\right|^{-a_{k}}\right)_{\bar{x}_{k}} \log \left|f_{k}\right| d \bar{x}_{k}\right. \\
+ & \left.\oint_{\left|x_{k}\right|=\epsilon} \log \left|x_{k}\right|^{-a_{k}}\left(\log \left|f_{k}\right|\right)_{x_{k}} d x_{k}+\int_{D_{k}(\epsilon)}\left(\log \left|f_{k}\right|\right)_{x_{k} \bar{x}_{k}} \log H_{k} d x_{k} \wedge d \bar{x}_{k}\right\}
\end{aligned}
$$

and, therefore,

$$
\int_{D_{k}(\epsilon)}\left(\log H_{k}\right)_{x_{k} \bar{x}_{k}} \log \left|f_{k}\right| \widehat{d x_{k}}=-\frac{a_{k} \pi}{2} \log \left|\mathbf{f}_{k}\right|+o(1)
$$

as $\epsilon \rightarrow 0$. Analogously

$$
\int_{D_{N+l}(\epsilon)}\left(\log H_{N+l}\right)_{y_{l} \bar{y}_{l}} \log \left|g_{l}\right| \widehat{d y}_{l}=-\frac{b_{l} \pi}{2} \log \left|\mathbf{g}_{l}\right|+o(1)
$$

as $\epsilon \rightarrow 0$.

On the other hand, the Alvarez formula implies that $\log \frac{\operatorname{det}\left(\Delta^{\mathbf{m}_{1}^{\epsilon}} \mid D_{k}(\epsilon)\right)}{\operatorname{det}\left(\Delta \mid D_{k}(\epsilon)\right)}=\frac{1}{3 \pi} \int_{D_{k}(\epsilon)}\left(\log H_{k}\right)_{x_{k} \bar{x}_{k}} \log H_{k} \widehat{d x_{k}}-\frac{a_{k}^{2}}{6} \log \epsilon+\frac{a_{k}}{3} \log \epsilon-\frac{a_{k}}{2}$ and

$$
\begin{aligned}
\log \frac{\operatorname{det}\left(\Delta^{\mathbf{m}_{2}^{\epsilon}} \mid D_{N+l}(\epsilon)\right)}{\operatorname{det}\left(\Delta \mid D_{N+l}(\epsilon)\right)}= & \frac{1}{3 \pi} \int_{D_{N+l}(\epsilon)}\left(\log H_{N+l}\right)_{y_{l} \bar{y}_{l}} \log H_{N+l} \widehat{d y_{l}} \\
& -\frac{b_{l}^{2}}{6} \log \epsilon+\frac{b_{l}}{3} \log \epsilon-\frac{b_{l}}{2},
\end{aligned}
$$

where $k=1, \ldots N, l=1, \ldots M$ and $\left(\Delta \mid D_{k}(\epsilon)\right)$ is the operator of the Dirichlet problem in the disk $D_{k}(\epsilon)$ for $\Delta=4 \partial_{z} \bar{\partial}_{z}$.

Thus, the right-hand side of (14) has the asymptotics

$$
\begin{gathered}
\frac{\operatorname{Area}\left(\mathcal{X}, \mathbf{m}_{1}\right)}{\operatorname{Area}\left(\mathcal{X}, \mathbf{m}_{2}\right)} \frac{\left\{\prod_{k=1}^{N} \operatorname{det}\left(\Delta^{\mathbf{m}_{1}} \mid D_{k}(\epsilon)\right)\right\}}{\left\{\prod_{l=1}^{M} \operatorname{det}\left(\Delta^{\mathbf{m}_{2}} \mid D_{N+l}(\epsilon)\right)\right\}} \frac{\left\{\prod_{l=1}^{M} \operatorname{det}\left(\Delta \mid D_{N+l}(\epsilon)\right)\right\}}{\left\{\prod_{k=1}^{N} \operatorname{det}\left(\Delta \mid D_{k}(\epsilon)\right)\right\}} \frac{\prod_{k=1}^{N}\left|\mathbf{f}_{k}\right|^{-a_{k} / 6}}{\prod_{l=1}^{M}\left|\mathbf{g}_{l}\right|^{-b_{l} / 6}} \\
\quad \times \epsilon^{\sum_{k=1}^{N}\left(\frac{a_{k}^{2}}{6}-\frac{a_{k}}{3}\right)-\sum_{l=1}^{M}\left(\frac{b_{l}^{2}}{6}-\frac{b_{l}}{3}\right)}(1+o(1))
\end{gathered}
$$

as $\epsilon \rightarrow 0$.

Due to [19], formula (28), one has

$$
\operatorname{det}\left(\Delta \mid D_{k}(\epsilon)\right)=\epsilon^{-1 / 3} \operatorname{det}\left(\Delta \mid D_{k}(1)\right)
$$

with

$$
\operatorname{det}\left(\Delta \mid D_{k}(1)\right)=2^{-1 / 6} \pi^{-1 / 2} \exp \left\{-2 \zeta^{\prime}(-1)-5 / 12\right\} .
$$

On the other hand, the disk $D_{k}$ with metric $\left|x_{k}\right|^{2 a_{k}}\left|d x_{k}\right|^{2}$ is isometric to the right circular cone $K\left(\epsilon, a_{k}\right)$ with the slant height $\frac{\epsilon^{a_{k}+1}}{a_{k}+1}$ and the angle $2 \pi\left(a_{k}+1\right)$ around the apex. Similarly to (6) it is easy to show that the value of the operator $\zeta$-function of the Laplace operator on this cone (with Dirichlet boundary conditions) at zero equals to $\frac{1}{12}\left(\frac{1}{a_{k}+1}+a_{k}+1\right)$ and, therefore,

$$
\operatorname{det}\left(\Delta^{\mathbf{m}_{1}} \mid D_{k}(\epsilon)\right)=\epsilon^{-\frac{1}{6}\left(a_{k}+1\right)\left(\frac{1}{a_{k}+1}+a_{k}+1\right)} \operatorname{det}\left(\Delta \mid K\left(1, a_{k}\right)\right) .
$$


Now sending $\epsilon$ to 0 in (18) and using (19), (21), one arrives at (11).

Remark 3. Notice that a rather lengthy expression for $\operatorname{det}\left(\Delta \mid K\left(1, a_{k}\right)\right)$ as a function of $a_{k}$ is given in (17], Theorem 1). This together with (19, 21) gives explicit expressions for the factors $C\left(a_{k}\right), C\left(b_{k}\right)$ from (11) which we do not reproduce here.

Remark 4. It is instructive to check that (11) agrees with the rescaling property (7).

The following simple lemma ("on three polyhedra") is a corollary of (11).

Lemma 1. Let $\mathcal{X}$ be a compact Riemann surface of an arbitrary genus $g$ and let $\mathbf{l}$, $\mathbf{m}$ and $\mathbf{n}$ be three conformal flat conical metrics on $\mathcal{X}$. Suppose that the metric $\mathbf{l}$ has conical points $P_{1}, \ldots, P_{L}$ with conical angles $2 \pi\left(a_{1}+1\right), \ldots, 2 \pi\left(a_{L}+1\right)$, the metric $\mathbf{m}$ has conical points $Q_{1}, \ldots, Q_{M}$ with conical angles $2 \pi\left(b_{1}+1\right), \ldots, 2 \pi\left(b_{M}+1\right)$ and the metric $\mathbf{n}$ has conical points $R_{1}, \ldots, R_{N}$ with conical angles $2 \pi\left(c_{1}+1\right), \ldots$, $2 \pi\left(c_{N}+1\right)$. (All the points $P_{l}, Q_{m}, R_{n}$ are supposed to be distinct.) Then one has the relation

$$
\prod_{i=1}^{N}\left[\frac{\mathbf{l}}{\mathbf{m}}\left(R_{i}\right)\right]^{c_{i}} \prod_{j=1}^{L}\left[\frac{\mathbf{m}}{\mathbf{n}}\left(P_{j}\right)\right]^{a_{j}} \prod_{k=1}^{M}\left[\frac{\mathbf{n}}{\mathbf{l}}\left(Q_{k}\right)\right]^{b_{k}}=1 .
$$

Proof. Rewrite the identity

using formula (11).

$$
\frac{\operatorname{det}^{*} \Delta^{1}}{\operatorname{det}^{*} \Delta^{\mathbf{m}}} \frac{\operatorname{det}^{*} \Delta^{\mathbf{m}}}{\operatorname{det}^{*} \Delta^{\mathbf{n}}} \frac{\operatorname{det}^{*} \Delta^{\mathbf{n}}}{\operatorname{det}^{*} \Delta^{\mathbf{l}}}=1
$$

Remark 5. The above lemma also follows from the Weil reciprocity law for harmonic functions with logarithmic singularities.

3.4. Flat surfaces with trivial holonomy and moduli spaces of holomorphic differentials on Riemann surfaces. We follow [12 and Zorich's survey [20]. Outside the vertices a Euclidean polyhedral surface $\mathcal{X}$ is locally isometric to a Euclidean plane, and one can define the parallel transport along paths on the punctured surface $\mathcal{X} \backslash\left\{P_{1}, \ldots, P_{N}\right\}$. The parallel transport along a homotopically nontrivial loop in $\mathcal{X} \backslash\left\{P_{1}, \ldots, P_{N}\right\}$ is generally nontrivial. If, e.g., a small loop encircles a conical point $P_{k}$ with conical angle $\beta_{k}$, then a tangent vector to $\mathcal{X}$ turns by $\beta_{k}$ after the parallel transport along this loop.

A Euclidean polyhedral surface $\mathcal{X}$ is called a surface with trivial holonomy if the parallel transport along any loop in $\mathcal{X} \backslash\left\{P_{1}, \ldots, P_{N}\right\}$ does not change tangent vectors to $\mathcal{X}$.

All conical points of a surface with trivial holonomy must have conical angles which are integer multiples of $2 \pi$.

A flat conical metric $g$ on a compact real oriented two-dimensional manifold $\mathcal{X}$ equips $\mathcal{X}$ with the structure of a compact Riemann surface. If this metric has trivial holonomy, then it necessarily has the form $g=|\omega|^{2}$, where $w$ is a holomorphic differential on the Riemann surface $\mathcal{X}$ (see 20]). The holomorphic differential $\omega$ has zeros at the conical points of the metric $g$. The multiplicity of the zero at the point $P_{m}$ with the conical angle $2 \pi\left(k_{m}+1\right)$ is equal to $k_{m} 2$

\footnotetext{
${ }^{2}$ There exist polyhedral surfaces with nontrivial holonomy whose conical angles are all integer multiples of $2 \pi$. To construct an example take a compact Riemann surface $\mathcal{X}$ of genus $g>1$ and choose $2 g-2$ points $P_{1}, \ldots, P_{2 g-2}$ on $\mathcal{X}$ in such a way that the divisor $P_{1}+\cdots+P_{2 g-2}$ is not
} 
The holomorphic differential $\omega$ is defined up to a unitary complex factor. This ambiguity can be avoided if the surface $\mathcal{X}$ is provided with a distinguished direction (see [20]), and it is assumed that $w$ is real along this distinguished direction. In what follows we always assume that surfaces with trivial holonomy are provided with such a direction.

Thus, to a Euclidean polyhedral surface of genus $g$ with trivial holonomy we put into correspondence a pair $(\mathcal{X}, \omega)$, where $\mathcal{X}$ is a compact Riemann surface and $\omega$ is a holomorphic differential on this surface. This means that we get an element of the moduli space, $\mathcal{H}_{g}$, of holomorphic differentials over Riemann surfaces of genus $g$ (see [12]).

The space $\mathcal{H}_{g}$ is stratified according to the multiplicities of zeros of $w$.

Denote by $\mathcal{H}_{g}(1, \ldots, 1)$ the stratum of $\mathcal{H}_{g}$, consisting of differentials $\omega$ with $2 g-2$ simple zeroes $P_{1}, \ldots, P_{2 g-2}$; the divisor of the differential $\omega$ is given by $(\omega)=\sum_{m=1}^{2 g-2} P_{m}$.

As in [6] introduce

- the following holomorphic multivalued $(g / 2,-g / 2)$-differential $\sigma(P, Q)$ :

$$
\sigma(P, Q)=\exp \left\{-\sum_{\alpha=1}^{g} \oint_{a_{\alpha}} v_{\alpha}(R) \log \frac{E(R, P)}{E(R, Q)}\right\} ;
$$

- the following holomorphic multivalued $g(1-g) / 2$-differential on $\mathcal{X}$ :

$$
\mathcal{C}(P)=\frac{1}{\mathcal{W}\left[v_{1}, \ldots, v_{g}\right](P)} \sum_{\alpha_{1}, \ldots, \alpha_{g}=1}^{g} \frac{\partial^{g} \Theta\left(K^{P}\right)}{\partial z_{\alpha_{1}} \ldots \partial z_{\alpha_{g}}} v_{\alpha_{1}} \ldots v_{\alpha_{g}}(P),
$$

where

$$
\mathcal{W}(P):=\operatorname{det}_{1 \leq \alpha, \beta \leq g}\left\|v_{\beta}^{(\alpha-1)}(P)\right\|
$$

is the Wronskian determinant of holomorphic differentials at the point $P$.

Denote by $\mathcal{A}_{P}(\cdot)$ the Abel map with the base point $P$. Then one has the relation

$$
\mathcal{A}((\omega))+2 K^{P}+\mathbb{B} \mathbf{r}+\mathbf{q}=0
$$

with some integer vectors $\mathbf{r}$ and $\mathbf{q}$. Let us emphasize that the vectors $\mathbf{r}, \mathbf{q}$ as well as the prime form and the differentials $C$ and $\sigma$ depend on the choice of the fundamental polygon $\widehat{\mathcal{X}}$.

The following theorem was proved in [10].

Theorem 2. Let a pair $(\mathcal{X}, \omega)$ be a point of the space $\mathcal{H}_{g}(1, \ldots, 1)$. Then the determinant of the Laplacian $\Delta^{|\omega|^{2}}$ is given by

$$
\operatorname{det}^{*} \Delta^{|\omega|^{2}}=\delta_{g} \operatorname{det} \Im \mathbf{B} \operatorname{Area}\left(\mathcal{X},|\omega|^{2}\right)\left|\tau_{g}(\mathcal{X}, \omega)\right|^{2},
$$

where $\delta_{g}$ is a constant depending only on the genus $g$ and $\tau_{g}(\mathcal{X}, \omega)$ is defined up to a unitary multiplicative factor (and not a choice of the fundamental polygon!) by the formula

$$
\tau_{g}^{-6}(\mathcal{X}, \omega)=e^{2 \pi i\left\langle\mathbf{r}, K^{P}\right\rangle} C^{-4}(P) \prod_{k=1}^{2 g-2} \sigma\left(P_{k}, P\right)\left\{E\left(P_{k}, P\right)\right\}^{(g-1)} .
$$

in the canonical class. Consider the flat conical conformal metric $\mathbf{m}$ corresponding to the divisor $P_{1}+\cdots+P_{2 g-2}$ according to the Troyanov theorem. This metric has nontrivial holonomy while all its conical angles are equal to $4 \pi$. 
Here $P$ is an arbitrary point of $\mathcal{X}$ and the integer vector $\mathbf{r}$ is defined by (26); the values of the prime form and $\sigma$ at the zeros $P_{k}$ of the differential $\omega$ are calculated in the local parameter $x_{k}(Q)=\sqrt{\int_{P_{k}}^{Q} \omega}$; the values of the prime form and $\sigma$ at the point $P$ are taken in the local parameter $z(Q)=\int^{Q} \omega$; the expression (28) is independent of the choice of $P$.

Remark 6. In 9] it was shown that the factor $\delta_{g}$ in (27) admits the representation

$$
\delta_{g}=(2 \pi)^{-4 / 3} \kappa_{0}^{g-1},
$$

where $\kappa_{0}$ is an absolute constant which could be expressed through spectral characteristics of some model operators.

\subsection{Determinant of the Laplacian on an arbitrary polyhedral surface of} genus $g>1$. From (11) and (27) follows the main result of the present paper:

Theorem 3. Let $\mathcal{X}$ be a compact Riemann surface of genus $g>1$ and let $\mathbf{m}$ be a conformal flat conical metric on $\mathcal{X}$ with conical points $P_{1}, \ldots, P_{N}$ with conical angles $2 \pi\left(a_{1}+1\right), \ldots, 2 \pi\left(a_{N}+1\right)$. Also let $\omega$ be a holomorphic one-form on $\mathcal{X}$ with $2 g-2$ simple zeros $Q_{1}, \ldots, Q_{2 g-2}$. Let $x_{k}$ be a distinguished local parameter for $\mathbf{m}$ near $P_{k}$ and $y_{l}$ be a distinguished local parameter for $\omega$ near $Q_{l}$. Introduce the functions $f_{k}, g_{l}$ and the complex numbers $\mathbf{f}_{\mathbf{k}}, \mathbf{g}_{\mathbf{l}}$ by

$$
\begin{aligned}
|\omega|^{2} & =\left|f_{k}\left(x_{k}\right)\right|^{2}\left|d x_{k}\right|^{2} \quad \text { near } P_{k}, \quad \mathbf{f}_{\mathbf{k}}:=f_{k}(0), \\
\mathbf{m} & =\left|g_{l}\left(y_{l}\right)\right|^{2}\left|d y_{l}\right|^{2} \quad \text { near } \quad Q_{l}, \quad \mathbf{g}_{\mathbf{l}}:=g_{l}(0) .
\end{aligned}
$$

Then

$$
\operatorname{det}^{*} \Delta^{\mathbf{m}}=\delta_{g} \frac{\prod_{k=1}^{M} C\left(a_{k}\right)}{C(1)^{2 g-2}} \operatorname{Area}(\mathcal{X}, \mathbf{m}) \operatorname{det} \Im \mathbf{B}\left|\tau_{g}(\mathcal{X}, \omega)\right|^{2} \frac{\prod_{l=1}^{2 g-2}\left|\mathbf{g}_{\mathbf{l}}\right|^{1 / 6}}{\prod_{k=1}^{N}\left|\mathbf{f}_{\mathbf{k}}\right|^{b_{k} / 6}},
$$

where $\tau_{g}(\mathcal{X}, \omega)$ is given by (28) $; C(a)$ and $\delta_{g}$ are described in Remarks 3 and 6 .

\section{ACKNOWLEDGEMEnTs}

The author would like to thank G. Carron and L. Hillairet for an important remark which led to a significant improvement of Proposition 1, he also acknowledges numerous useful conversations with D. Korotkin. This work was written during the stay of the author at the Hausdorff Research Institute for Mathematics. The author thanks the institute for the hospitality and support. The research of the author was also supported by NSERC.

\section{REFERENCES}

[1] Alvarez, O., Theory of strings with boundaries, Nucl. Phys. B216, 125 (1983). MR701643 (85e:81095)

[2] Aurell, E., Salomonson, P., On functional determinants of Laplacians in polygons and simplicial complexes, Communications in Mathematical Physics, Volume 165, Number 2, 1994, 233-259. MR 1301847 (95i:58186)

[3] Burghelea, D., Friedlander, L., and Kappeler, T., Meyer-Vietoris type formula for determinants of elliptic differential operators, J. of Funct. Anal., 107, 34-65 (1992). MR.1165865 (93f:58242)

[4] Cheeger, J., Spectral geometry of singular Riemannian spaces, J. Diff. Geometry, 18 (1983), 575-657. MR730920 (85d:58083)

[5] Fay, John D., Theta-functions on Riemann surfaces, Lect. Notes in Math., 352, Springer (1973). MR0335789(49:569) 
[6] Fay, John D., Kernel functions, analytic torsion, and moduli spaces, Memoirs of the AMS 464 (1992). MR 1146600 (93e:58192)

[7] King, Hala Khuri, Determinants of Laplacians on the space of conical metrics on the sphere, Transactions of AMS, 339, 525-536 (1993). MR1102890 (93m:58118)

[8] Klochko, Yu, Kokotov, A., Genus one polyhedral surfaces, spaces of quadratic differentials on tori and determinants of Laplacians, Manuscripta Mathematica, 122, 195-216 (2007). MR2295112(2008c:58024)

[9] Kokotov, A., On the asymptotics of determinant of Laplacian at the principal boundary of the principal stratum of the moduli space of Abelian differentials, to appear in Transactions of AMS.

[10] Kokotov, A., Korotkin, D., Tau-functions on spaces of Abelian differentials and higher genus generalization of Ray-Singer formula, Journal of Differential Geometry, 82 (2009), 35-100. MR2504770(2010c:58041)

[11] Kokotov, A., Korotkin, D., Tau-functions on the spaces of Abelian and quadratic differentials and determinants of Laplacians in Strebel metrics of finite volume, preprint of Max-Planck Institute for Mathematics in the Science, Leipzig, 46/2004; math.SP/0405042.

[12] Kontsevich, M., Zorich, A., Connected components of the moduli spaces of holomorphic differentials with prescribed singularities, Invent. Math., 153, 631-678 (2003). MR2000471 (2005b:32030)

[13] Loya, P., Mcdonald, P., Park, J., Zeta regularized determinants for conic manifolds, Journal of Functional Analysis, 242 (2007), N1, 195-229. MR2274020 (2007g:58036)

[14] McKean, H. P., Singer, I. M., Curvature and the eigenvalues of the Laplacian, J. Diff. Geometry, 1 (1967), 43-69. MR0217739 (36:828)

[15] Osgood, B., Phillips, R., Sarnak, P., Extremals of determinants of Laplacians, Journal of Functional Analysis, Vol. 80, N1, 148-211 (1988). MR960228 (90d:58159)

[16] Ray, D. B., Singer, I. M., Analytic torsion for complex manifolds, Ann. of Math. (2) 98 (1973), N1, 154-177. MR0383463 (52:4344)

[17] Spreafico, M., Zeta function and regularized determinant on a disk and on a cone, Journal of Geometry and Physics, 54 (2005), 355-371. MR2139088(2005k:11184)

[18] Troyanov, M., Les surfaces euclidiennes à singularités coniques, Enseign. Math. (2), 32 (1986), 79-94. MR850552 (87i:30079)

[19] Weisberger, W., Conformal invariants for determinants of Laplacians on Riemann surfaces, Commun. Math. Phys., 112, 633-638 (1987). MR910583 (89c:58135)

[20] Zorich, A., Flat Surfaces, in collection "Frontiers in Number Theory, Physics and Geometry. Vol. 1: On random matrices, zeta functions, and dynamical systems", P. Cartier, B. Julia, P. Moussa, P. Vanhove (Eds.), Springer-Verlag, Berlin, 2006, 439-586. MR2261104 (2007i:37070)

Department of Mathematics and Statistics, Concordia University, 1455 de Maisonneuve Boulevard West, Montreal, Quebec, H3G 1M8 Canada

E-mail address: alexey@mathstat.concordia.ca 